\title{
Effects of different routes of tirofiban injection on the left ventricular function and prognosis of patients with myocardial infarction treated with percutaneous coronary intervention
}

\author{
CUIHUA ZHAO ${ }^{1,2},{\text { GUANCHANG } \text { CHENG }^{1} \text {, RUILI HE }}^{1}$, HONGYU GUO $^{1}$, \\ YANMING $\mathrm{LI}^{1}$, XUELI LU ${ }^{1}$, YUAN ZHANG ${ }^{1}$ and CHUNGUANG QIU ${ }^{2}$ \\ ${ }^{1}$ Department of Cardiology, Henan University Huaihe Hospital, Kaifeng, Henan 475000; \\ ${ }^{2}$ Department of Cardiology, The First Affiliated Hospital of Zhengzhou University, Zhengzhou, Henan 450052, P.R. China
}

Received July 7, 2014; Accepted March 17, 2015

DOI: 10.3892/etm.2015.2401

\begin{abstract}
The aim of this study was to investigate the effect of different routes of tirofiban injection on the function of the left ventricle and the prognosis of patients with myocardial infarction (MI) treated with percutaneous coronary intervention (PCI). Ninety-five patients with MI treated with PCI were divided into two groups [coronary $(n=49)$ and intravenous $(n=46)$ ] according to the injection route. A comparison of the left ventricular function and prognosis was made between the two groups following PCI. The success rate of PCI in the coronary group was $97.96 \%$, which was higher than that in the intravenous group $(\mathrm{P}<0.05)$. No significant differences were identified in the platelet count (PLT) and platelet aggregation rate (PAR) between the two groups prior to the tirofiban injection. Following the tirofiban injection, the PLT decreased markedly in both groups, with no significant differences between them. The PAR also decreased significantly in the two groups; however, the value in the coronary group was lower than that in the intravenous group $(\mathrm{P}<0.05)$. The improvements in the thrombolysis in MI grades, left ventricular ejection fraction and left ventricular diastolic function were greater in the coronary group than those in the intravenous group $(\mathrm{P}<0.05)$. All patients received follow-up for 30 days and the incidence of bleeding in the coronary group was lower than that in the intravenous group $(\mathrm{P}<0.05)$. No significant differences were recorded in the recurrence rates of MI, arrhythmia, myocardial ischemia, thrombocytopenia and mortality between the two groups. In conclusion, the administration of tirofiban into the coronary artery could effectively improve the blood flow, left ventricular function and prognosis of patients with MI treated with PCI.
\end{abstract}

Correspondence to: Dr Chunguang Qiu, Department of Cardiology, The First Affiliated Hospital of Zhengzhou University, 1 East Jianshe Road, Zhengzhou, Henan 450052, P.R. China

E-mail: chunguangqiucn@yeah.net

Key words: tirofiban, percutaneous coronary intervention, myocardial infarction, left ventricle function, prognosis

\section{Introduction}

Percutaneous coronary intervention (PCI), also known as coronary angioplasty, is a nonsurgical technique for treating obstructive coronary artery disease (CAD), including unstable angina and multivessel CAD. PCI is the main treatment for patients with acute myocardial infarction (MI), since it can improve myocardial reperfusion and induce coronary artery recanalization (1). PCI can effectively decrease the mortality rate of patients with MI; however, some patients have exhibited hemodynamic instability following PCI treatment, and this instability can aggravate the ischemic necrosis of cardiac muscle cells and increase the incidence of arrhythmia and heart failure (2). The most commonly used treatment for hemodynamic instability is the arterial injection of verapamil, adenosine or nitroglycerin; however, the effects of these treatments are poor (3).

Excessive platelet activation and aggregation plays a pivotal role in the disease progression in patients with MI following PCI. Glycoprotein IIb/IIIa is a key factor influencing thrombosis and platelet aggregation (4). A recent study showed that glycoprotein IIb/IIIa antagonists could improve myocardial perfusion and clinical outcomes by inhibiting platelet aggregation (5). Tirofiban, as an antagonist of glycoprotein IIb/IIIa, is considered to be one of the most effective drugs in inhibiting platelet aggregation (6). The commonly used administration routes of tirofiban are intravenous and intra-arterial injection. It has been suggested that the administration of tirofiban by intra-arterial injection could promote drug absorption in the diseased region and enhance the inhibition of platelet aggregation (7). The present study investigated the effects of the two different methods of tirofiban administration on the function of the left ventricle as well as on the prognosis of patients with MI treated with PCI.

\section{Subjects and methods}

Subjects. Ninety-five patients that had been admitted to the Department of Cardiology in the First Affiliated Hospital of Zhengzhou University (Zhengzhou, Henan, China) between January 2011 and March 2014 with the clinical diagnosis 
Table I. Baseline characteristics of the patients.

\begin{tabular}{|c|c|c|c|c|}
\hline Characteristics & $\begin{array}{l}\text { Intravenous } \\
\text { group, } n=46\end{array}$ & $\begin{array}{l}\text { Coronary } \\
\text { group, } n=49\end{array}$ & $\mathrm{~T} / \chi^{2}$ & P-value \\
\hline \multicolumn{5}{|l|}{ Gender } \\
\hline Male & 32 & 33 & 1.19 & $>0.05$ \\
\hline Female & 14 & 16 & & \\
\hline \multicolumn{5}{|l|}{ Age (years) } \\
\hline Range & $54-81$ & $53-78$ & 1.35 & $>0.05$ \\
\hline Mean & $64.34 \pm 4.21$ & $63.27 \pm 5.07$ & & \\
\hline \multicolumn{5}{|l|}{ Complications (n) } \\
\hline Hypertension & 18 & 19 & 1.10 & $>0.05$ \\
\hline Diabetes & 17 & 16 & 1.10 & $>0.05$ \\
\hline Hyperlipidemia & 10 & 12 & 1.24 & $>0.05$ \\
\hline \multicolumn{5}{|l|}{$\mathrm{PCI}$ treatment region $(\mathrm{n})$} \\
\hline Left anterior descending branch & 23 & 25 & 1.34 & $>0.05$ \\
\hline Left circumflex artery & 9 & 10 & & \\
\hline Right coronary artery & 14 & 15 & & \\
\hline Time from onset of MI to surgery (h) & $5.81 \pm 0.67$ & $5.92 \pm 0.76$ & 1.21 & $>0.05$ \\
\hline Number of interventional stents & $1.83 \pm 0.57$ & $1.79 \pm 0.61$ & 1.17 & $>0.05$ \\
\hline
\end{tabular}

PCI, percutaneous coronary intervention; MI, myocardial infarction.

of MI were enrolled in the study (8). PCI was performed $12 \mathrm{~h}$ after the onset of acute MI. The following exclusion criteria were used: i) Aneurysm, ii) arteriovenous malformation, iii) intracranial tumor, iv) intracranial hemorrhage, v) platelet count (PLT) $\leq 100 \times 10^{9} / 1$, vi) cardiogenic shock, vii) enterorrhagia, viii) allergy to tirofiban and ix) pregnancy/lactation in women. The patients were divided into two groups according to the method used to administer the loading-dose of tirofiban: Coronary $(n=49)$ and intravenous $(n=46)$. No significant differences were observed between the two groups in the baseline characteristics such as gender, age, complications, PCI treatment region, number of interventional stents and time interval from the onset of MI to surgery (Table I). The present study was conducted in accordance with the Declaration of Helsinki and was approved by the Ethics Committee of the First Affiliated Hospital of Zhengzhou University. Written informed consent was obtained from all patients.

Treatment methods. Patients in the two groups received oral administration of $300 \mathrm{mg}$ aspirin (Bayer AG, Leverkusen, Germany; batch no. J20080078), 300 mg clopidogrel (Sanofi-Aventis, Paris, France; batch no. J20130083) and an injection of 8,000 units heparin (Wanbang Biopharmaceuticals, Xuzhou, China; batch no. H32020612) prior to the PCI treatment. The patients from the intravenous group then received an intravenous injection of $10 \mu \mathrm{g} / \mathrm{kg}$ tirofiban (Grand Pharmaceutical Group, Wuhan, China; batch no. H20041165) while those from the coronary group received an intracoronary injection of tirofiban (also $10 \mu \mathrm{g} / \mathrm{kg}$ ). Tirofiban was then infused at a dose of $0.15 \mu \mathrm{g} / \mathrm{kg} / \mathrm{min}$ for 24-36 $\mathrm{h}$ after PCI to maintain a steady dose.
Observation indexes. The evaluation standards for PCI success were as follows: Good stent apposition, no coronary artery dissection, no vascular occlusion, no thrombosis, no clinical adverse events and residual stenosis $<20 \%$. The efficacy of tirofiban was assessed using the platelet aggregation rate (PAR) and was inversely proportional to the PAR value. The PAR was measured using the AggRAM ${ }^{\text {TM }}$ system (Helena Biosciences Europe, Gateshead, UK).

The thrombolysis in MI (TIMI) grades were used to assess the forward blood flow and were graded as follows: Grade 0 , no blood perfusion in the infarction-related artery and occlusion of distal vessels without blood flow; grade 1, distal stenosis of the coronary artery without blood flow; grade 2 , distal stenosis of the coronary artery with blood flow, but complete filling is slow; grade 3, blood flow is similar to normal coronary artery blood flow. TIMI grades 2 and 3 suggested an improved blood flow and, therefore, a better prognosis (9).

The assessment of left ventricular function was performed by echocardiography 1 month after surgery. The left ventricular ejection fraction (LVEF) was used to assess the left ventricular systolic function and was proportional to systolic function. The ratio of the early to late ventricular filling velocities (E/A value) was used to assess the left ventricular diastolic function, and an E/A $>1$ suggested a better diastolic function.

The patients were followed up for 30 days after PCI and the recurrence of MI, arrhythmia, myocardial ischemia, minor bleeding, thrombocytopenia and mortality in the two groups was assessed. Minor bleeding was diagnosed if the drop in hemoglobin was 30-50 g/l. Thrombocytopenia was defined as PLT $<100 \times 10^{9} / 1$. 
Table II. Comparison of TIMI flow grades between the 2 groups.

TIMI flow grades, n $(\%)$

\begin{tabular}{lccccc}
\cline { 2 - 5 } Groups & Cases, $\mathrm{n}$ & Grade 0 & Grade 1 & Grade 2 & Grade 3 \\
\hline Intravenous & 46 & $0(0)$ & $3(6.52)$ & $17(36.96)$ & $26(56.52)$ \\
Coronary & 49 & $0(0)$ & $0(0)$ & $12(24.49)$ & $37(75.51)$ \\
$\chi^{2}$ & & 0.00 & 2.03 & 4.06 & 5.04 \\
P-value & $>0.05$ & $>0.05$ & $<0.05$ & $<0.05$
\end{tabular}

TIMI, thrombolysis in myocardial infarction.

Table III. Comparison of left ventricular function between the 2 groups.

\begin{tabular}{|c|c|c|c|c|}
\hline \multirow[b]{2}{*}{ Groups } & \multirow[b]{2}{*}{ Cases, $\mathrm{n}$} & \multirow[b]{2}{*}{ LVEF (\%) } & \multicolumn{2}{|c|}{ E/A value, n (\%) } \\
\hline & & & $>1$ & $\leq 1$ \\
\hline Intravenous & 46 & $54.19 \pm 4.37$ & $13(28.26)$ & $33(71.74)$ \\
\hline Coronary & 49 & $63.58 \pm 4.52$ & $26(53.06)$ & $23(46.94)$ \\
\hline$T / \chi^{2}$ & & 3.62 & 5.73 & 6.89 \\
\hline P-value & & $<0.05$ & $<0.05$ & $<0.05$ \\
\hline
\end{tabular}

LVEF, left ventricular ejection fraction; E/A value, ratio of the early to late ventricular filling velocities.

Table IV. Incidence of adverse clinical events in the 2 groups.

\begin{tabular}{lcccccrr}
\hline Groups & Cases, $\mathrm{n}$ & $\begin{array}{c}\text { Recurrence } \\
\text { of MI, n (\%) }\end{array}$ & $\begin{array}{c}\text { Arrhythmia, } \\
\mathrm{n}(\%)\end{array}$ & $\begin{array}{c}\text { Myocardial } \\
\text { ischemia, n(\%) }\end{array}$ & $\begin{array}{c}\text { Minor } \\
\text { bleeding, }(\%)\end{array}$ & $\begin{array}{c}\text { Thrombocytopenia, } \\
\mathrm{n}(\%)\end{array}$ & $\begin{array}{c}\text { Mortality, } \\
\mathrm{n}(\%)\end{array}$ \\
\hline Intravenous & 46 & $0(0)$ & $3(6.52)$ & $4(8.70)$ & $10(21.74)$ & $0(0)$ & $0(0)$ \\
Coronary & 49 & $0(0)$ & $3(6.12)$ & $4(8.16)$ & $5(10.20)$ & $0(0)$ & $0(0)$ \\
$\chi^{2}$ & & 0.00 & 1.06 & 1.30 & 4.09 & 0.00 & 0.00 \\
P-value & $>0.05$ & $>0.05$ & $>0.05$ & $<0.05$ & $>0.05$ & $>0.05$ \\
\hline
\end{tabular}

MI, myocardial infraction.
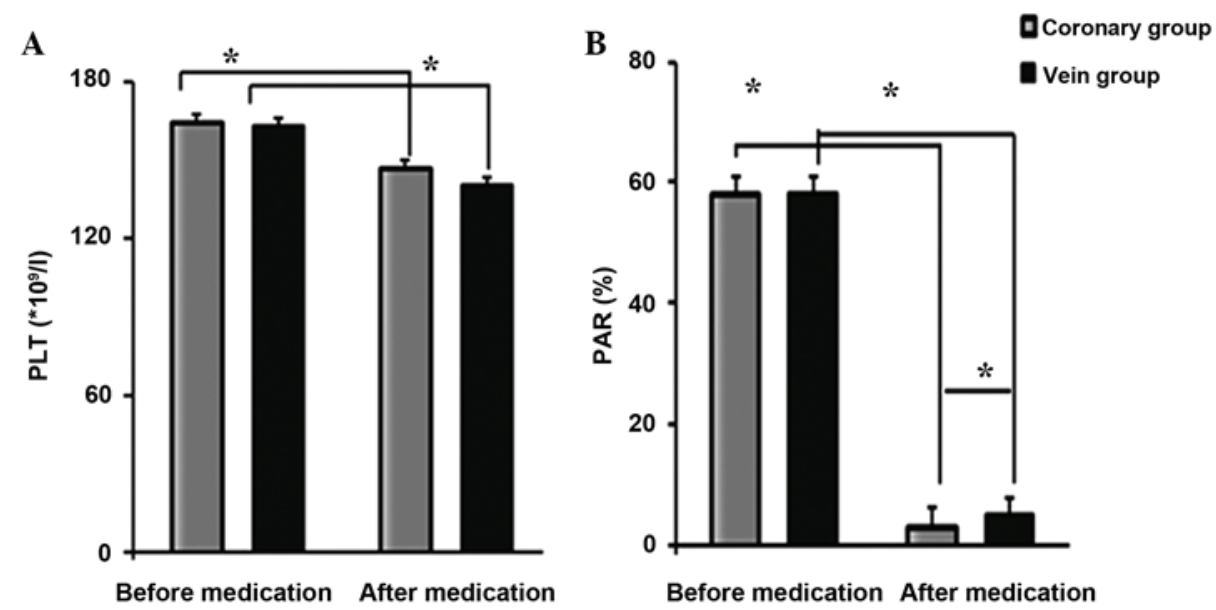

Figure 1. Comparison of (A) PLT and (B) PAR between the 2 groups. " $\mathrm{P}<0.05$. Data are presented as the mean \pm standard deviation. PLT, platelet count; PAR, platelet aggregation rate. 
Statistical analysis. All data were analyzed using SPSS 18.0 software (SPSS Inc., Chicago, IL, USA) and are expressed as the mean \pm standard deviation. The data were analyzed using the unpaired Student's t-test to compare the two groups and the $\chi^{2}$ test. $\mathrm{P}<0.05$ was considered to indicate a statistically significant difference.

\section{Results}

Clinical outcomes. The success rates of PCI in the intravenous and coronary groups were 86.96 and $97.96 \%$, respectively, with significant difference between the groups $\left(\chi^{2}=3.91\right.$, $\mathrm{P}<0.05)$. Prior to the tirofiban injection, no significant difference was recorded in the PLT between the two groups (Fig. 1). Following the tirofiban injection, the PLT decreased markedly in both groups $(\mathrm{T}=2.68, \mathrm{P}<0.05)$ but there remained no significant difference between them ( $\mathrm{T}=2.07, \mathrm{P}>0.05)$. Prior to the tirofiban injection, no significant difference was identified in the PAR between the two groups ( $\mathrm{T}=1.02, \mathrm{P}>0.05)$; however, 10 min after the tirofiban injection, the PAR in the coronary group was significantly lower than that in the intravenous group ( $\mathrm{T}=2.68, \mathrm{P}<0.05)$, although marked decreases were observed in both groups. As shown in Table II, the proportion of patients with TIMI grades 2 and 3 in the coronary group was higher than that in the intravenous group $(\mathrm{P}<0.05)$.

Left ventricular function. As shown in Table III, the LVEF and E/A value of the coronary group improved significantly compared with the same value in the intravenous group $(\mathrm{P}<0.05)$.

Prognosis. The incidence of bleeding in the coronary group was considerably lower than that in the intravenous group $(\mathrm{P}<0.05)$. As shown in Table IV, no significant difference was found in the incidence of adverse clinical events such as recurrence of MI, arrhythmia, myocardial ischemia, thrombocytopenia and mortality between the two groups $(\mathrm{P}>0.05)$.

\section{Discussion}

PCI is an effective treatment for acute MI. Slow blood flow is one of the main factors affecting the prognosis of patients with MI treated with PCI. Slow flow refers to the recanalization of the vasculature without effective reperfusion in the myocardial cells. The incidence of slow flow in patients with MI treated by PCI has been reported to be $18 \%$, with this slow flow being caused by distal-end thrombosis during PCI due to the atherosclerotic plaque or thrombosis $(10,11)$. Antiplatelet therapy is the key factor in ensuring the success of the PCI and effective reperfusion in patients with MI (12). Aspirin, a commonly used antiplatelet drug, is said to be capable of decreasing thrombosis by inhibiting cyclooxygenase (13), while clopidogrel prevents platelet aggregation by inhibiting adenosine diphosphate receptor expression and activity on the platelet surface (14); however, clinical research has shown that neither aspirin nor clopidogrel on their own can effectively improve the slow blood flow of patients with MI (15). Therefore, enhancing antiplatelet therapy could play a crucial role in improving the prognosis, heart function and reperfusion in patients with MI treated with PCI. The involvement of glycoprotein IIb/IIIa receptors in platelet aggregation suggests that glycoprotein IIb/IIIa antagonists could be used to effectively prevent platelet aggregation $(16,17)$. In the present study, a comparison was made between the effects of different routes of tirofiban administration on the function of the left ventricle and the prognosis of patients with MI treated with PCI, based on the premise of aspirin and clopidogrel administration prior to PCI.

The present study demonstrated that intra-arterial administration of tirofiban could increase the success rate of PCI (97.96 vs. $86.96 \%$ ) compared with intravenous administration. Following the tirofiban injection, the PLT dropped to $145 \times 10^{9} / 1$ in both groups, which did not meet the diagnostic criteria for thrombocytopenia, suggesting drug safety, while the PAR was lower in the coronary group than that in the intravenous group, suggesting a high level of drug absorption and an enhanced ability to inhibit platelet aggregation in the coronary group. The TIMI grade, LVEF and E/A of the coronary group showed greater improvements than those of the intravenous group. In addition, the incidence of minor bleeding was lower in the coronary group than that in the intravenous group. No significant differences were identified in the recurrence rates of MI, arrhythmia, myocardial ischemia, thrombocytopenia and mortality between the two groups. As reported, tirofiban could decrease the PAR and thrombosis and improve the microcirculation and reperfusion of myocardial cells by antagonizing glycoprotein IIb/IIIa receptors (18-21). In conclusion, intra-arterial administration of tirofiban could effectively result in an improvement in the blood flow, left ventricular function and prognosis of patients with MI treated with PCI by inhibiting platelet aggregation.

\section{References}

1. Niccoli G, Kharbanda RK, Crea F and Banning AP: No-reflow: Again prevention is better than treatment. Eur Heart J 31: 2449-2455, 2010.

2. Zhang D, Wang L, Du J, et al: Effect of intracoronary tirofiban combined with nitroprusside injection through thrombus aspiration catheter during primary percutaneous coronary intervention on acute anterior myocardial infarction patients with heavy thrombosis burden. Zhonghua Xin Xue Guan Bing Za Zhi 42: 25-30, 2014 (In Chinese).

3. Li MW, Zhao XM, Rao LX, et al: The clinical efficacy and safety of fondaparinux combined with tirofiban hydrochloride in patients with acute coronary syndrome undergoing complex percutaneous coronary intervention. Zhonghua Nei Ke Za Zhi 52: 1037-1040, 2013 (In Chinese).

4. Ren LH, Peng JJ, Ye HM, Wang ZY and Chen C: High-dose tirofiban in patients with acute ST-segment elevation myocardial infarction undergoing primary percutaneous coronary interventio. Zhonghua Yi Xue Za Zhi 92: 1981-1983,2012 (In Chinese).

5. Li X, Lei Y and Zheng Q: Myocardial infarction caused by coronary artery dissection due to blunt injury: Is thromboaspiration an appropriate treatment. Hellenic J Cardiol 55: 61-64, 2014.

6. He WK, Li MY, Xing XW and Lu DF: The effects of different ways of tirofiban injection on prognosis of the patients with AIM treated with PIC. Shi Yong Yi Xue Za Zhi 28: 2936-2939, 2012.

7. Tong ZC, Li Q, Chen M, et al: Efficacy comparison of combined intracoronary administration of high-dose adenosine and tirofiban versus intracoronary tirofiban during primary percutaneous coronary intervention in patients with acute myocardial infarction. Zhonghua Xin Xue Guan Bing Za Zhi 41: 839-844, 2013 (In Chinese).

8. Zhang Y, Gao CY, Zhu ZY, et al: Efficacy and safety of tirofiban use after successful percutaneous coronary intervention for patients with moderate to high risk non-ST segment elevation acute coronary syndromes. Zhonghua Xin Xue Guan Bing Za Zhi 41: 731-735, 2013 (In Chinese). 
9. Gibson CM, Karha J, Giugliano RP, et al; INTEGRITI Study Group: Association of the timing of ST-segment resolution with TIMI myocardial perfusion grade in acute myocardial infarction. Am Heart J 147: 847-852, 2004

10. Qin T, Xie L and Chen MH: Meta-analysis of randomized controlled trials on the efficacy and safety of intracoronary administration of tirofiban for no-reflow phenomenon. BMC Cardiovasc Disord 13: 68, 2013.

11. Li W, Fu X, Xue H, et al: Efficacy and safety of standard-dose versus half-dose tirofiban in patients with non-ST elevation acute coronary syndromes undergoing early percutaneous coronary intervention. Cardiovasc Ther 31: 210-214, 2013.

12. Zhao YJ, Fu XH, Ma XX, et al: Intracoronary fixed dose of nitroprusside via thrombus aspiration catheter for the prevention of the no-reflow phenomenon following primary percutaneous coronary intervention in acute myocardial infarction. Exp Ther Med 6: 479-484, 2013.

13. Balghith M, Al-Ghamdi A, Zain el $\mathrm{H}$ and Al-Saileek A: Intracoronary Reopro During Percutaneous Coronary Intervention In Acute And Stable Patient Can Influence Stent Thrombosis Formation (IRPASST) study. Heart Views 14: 62-67, 2013.

14. Tas MH, Simsek Z, Ayan A, et al: Effects of tirofiban maintenance duration on myocardial perfusion defect severity in anterior myocardial infarction. Adv Ther 30: 834-844, 2013

15. Hong TT, Huang J, Driscoll E and Lucchesi BR: The antithrombotic effect of melagatran in combination with clopidogrel and/or aspirin (carotid artery primary thrombosis study). J Cardiovasc Pharmacol 46: 526-533, 2005.
16. Zhang Y, Gao C, Liu H, et al: Routine early versus deferred provisional tirofiban treatment in patients with acute coronary syndrome undergoing percutaneous coronary intervention. Clin Exp Pharmacol Physiol 40: 289-294, 2013.

17. Deshpande NV, Pratiti R, Admane P, Mukherjee D and Mardikar HM: Safety and efficacy of bivalirudin with glycoprotein IIb/IIIa for high-risk percutaneous coronary intervention. Indian Heart J 64: 444-448, 2012.

18. Balghith MA: High bolus tirofiban vs abciximab in acute STEMI patients undergoing primary PCI - The Tamip study. Heart Views 13: 85-90, 2012.

19. Liu X, Dong P, Xing S, et al: Clinical evaluation of thrombus aspiration combined with tirofiban in patients with acute myocardial infarction with elective percutaneous coronary intervention. J Int Med Res 41: 1532-1540, 2013.

20. Jia Z, Guo M, Zhang YQ, Liang HQ and Song Y: Short-term effect of upstream administration in comparison to deferred injection of tirofiban on patients with acute ST-segment elevation myocardial infarction undergoing primary percutaneous coronary intervention. J Interv Cardiol 26: 332-329, 2013.

21. Zhang Q, Qiu JP, Zhang RY, et al: Improved outcomes from transradial over transfemoral access in primary percutaneous coronary intervention for patients with acute ST-segment elevation myocardial infarction and upstream use of tirofiban. Chin Med J (Engl) 126: 1063-1068, 2013. 